\title{
Necesidades de entrenamiento del personal basado en el enfoque de competencias. Estudio de un caso
}

\author{
Revilla Delgado, Noleyda del Valle* \\ Acosta Campos, Ivonne Cristina** \\ Marval Galvis, Elina Victoria***
}

\section{Resumen}

El objetivo de la investigación fue detectar las necesidades de entrenamiento del personal administrativo que ocupa cargo profesional en el área de finanzas de la Universidad Nacional Experimental Rafael María Baralt con base en el enfoque de competencias. El estudio es descriptivo. Las técnicas de recolección de datos fueron la encuesta y la revisión documental. La población fue de 49 personas, a quienes se le aplicó el instrumento Detección de Necesidades de Entrenamiento por Competencias, con 58 ítems. Los resultados indicaron que los Grupos Contaduría y Presupuesto tienen un grado de necesidad de entrenamiento mediano para las competencias: genéricas, laborales y básicas. Los Grupos Administración y Control Interno y Auditoría tienen un grado de necesidad mediano en el desarrollo de competencias tanto genéricas como básicas, y bajo en las laborales. El Grupo Análisis y Control Financiero posee un grado de necesidad bajo en las genéricas y laborales, y mediano en las básicas. Los datos obtenidos implican un cambio en la manera como la Universidad ha llevado a cabo el proceso de entrenamiento; asimismo, los profesionales del área de finanzas al conocer las carencias en las competencias detectadas, pueden emprender un proceso de desaprender y aprender hábitos de pensamientos, sentimientos y acción a manera de autoevaluar su desempeño.

Palabras clave: Necesidades de entrenamiento, competencias, manual de cargos.

\section{Recibido: 11-10-07 Aceptado: 25-06-08}

* M.Sc. en Gerencia de Recursos Humanos, Administradora. Programa Administración de la UNERMB. E-mail: noleydadelvalle@cantv.net.

** Dra. en Ciencias, mención Gerencia. Prof. Titular de la UNERMB. Coordinadora de Investigación del Postgrado de la UNERMB. Investigadora acreditada ante el PPI. Adscrita al Programa de Investigación PROINEE. Prof. meritoria CONABA, Nivel II. E-mail: acostai@cantv.net.

*** Dra. en Ciencias de la Educación. Prof. Titular del Postgrado de la UNERMB. Investigadora acreditada ante el PPI. Adscrita al Programa de Investigación PROINEE. Coordinadora de línea de investigación. Prof. meritoria CONABA, Nivel I. E-mail: elinavictoria@cantv.net. 


\title{
Training Needs for Personnel Based on the Competence Focus. A Case Study
}

\begin{abstract}
The objective of this investigation was to detect the training needs of administrative personnel who occupy a professional position in the finance area at the National Experimental University Rafael María Baralt, based on the competences focus. The study is descriptive. Data collection techniques were the interview and documentary review. The population consisted of 49 people, to whom the instrument "Detection of Training Needs by Competences," consisting of 58 items, was applied. Results indicated that the Groups Accounting and Budget have a medium degree of need for training in generic, labor-related and basic competences. The Groups Administration and Internal Control and Auditing have a medium degree of need for developing basic competences and are low in those related to labor. The Group Financial Analysis and Control has a low degree of need in generic and labor-related competences, and a medium need for the basic. The data obtained implies a change in the way the University carries out the training process; likewise, the financial area professionals, on learning about the deficiencies detected regarding the competences, can undertake a process of unlearning and learning new habits of thought, feelings and action in order to evaluate their own performance.
\end{abstract}

Key words: Training needs, competences, position manual.

\section{Introducción}

El talento humano se muestra como un factor determinante para que las organizaciones tanto públicas como privadas alcancen una posición competitiva en el mercado, como resultado de la incidencia que tienen en aspectos tales como: capacidad innovadora, productividad, calidad de los productos y servicios.

Los avances científicos, tecnológicos y humanísticos manifestados de manera acelerada en el ámbito mundial, han exigido cambios en la organización social del trabajo. Esto ha llevado a las unidades productivas a desplazarse de una organización jerárquica piramidal y estructura ocupacional segmentada a una organización sistemática basada en el trabajo en equipo multifuncional, polivalente e interdisciplinario.
Esa exigencia implica cambios fundamentales en los mecanismos utilizados en la selección y capacitación del personal. Por ello es necesario que las organizaciones, entre éstas las instituciones educativas de educación superior, le otorguen la importancia que tiene su gente, adopten políticas y estrategias para obtener el máximo provecho de su personal, preparándolo para asumir los retos presentes y futuros, lo cual puede lograrse a través de una gestión efectiva de su capital humano, con una verdadera práctica de administración de personal, orientada a procesos tan importantes como el entrenamiento.

Con base en esas afirmaciones, la planificación del talento humano se orienta a entrenar al personal de acuerdo al enfoque de competencias, para que potencien sus capacidades, habilidades, 
destrezas, procesos de pensamiento, entre otros aspectos, de manera holística.

Es importante resaltar que las organizaciones persiguen, el alto rendimiento y efectividad en sus procesos de producción y servicio, así como responder a las cambiantes tendencias y exigencias de la sociedad, por lo cual se requiere determinar las competencias del personal que allí labora. En las organizaciones educativas es imprescindible considerar las competencias, ya que permiten identificar las potencialidades del personal que ejerce el cargo, así como de quienes aspiren ingresar. Esto conlleva a reconocer las características requeridas por el puesto, no sólo en función de las tareas a realizar, sino también de los aspectos personales, sociales y técnicos inherentes al cargo.

Con relación a lo expuesto, la investigación que se presenta, cuyo propósito es detectar las necesidades de entrenamiento del personal administrativo de la Universidad Nacional Experimental "Rafael María Baralt" con base en el enfoque de competencias, pretende dar respuesta a la situación planteada.

\section{Contextualización de la temática}

Venezuela al igual que el resto de los países de América Latina y del mundo, está inmersa en un entorno caracterizado por profundos cambios en lo económico, político, social y cultural. Las fronteras entre los países se debilitan y el paradigma aceptado es el de la apertura comercial y la competencia global. La Organización de la Naciones Unidas para la Educación, la Ciencia y la Cultura
(UNESCO), al presentar el Programa 21 para la educación superior, con el objeto de promover el desarrollo sostenible de los países; expresó que la presente etapa de la historia del mundo se caracteriza por una mutación fundamentalmente diferente de las experimentadas en el pasado, entendida ésta como las alteraciones producidas en las estructuras. De igual manera, la Comisión Nacional de Currículo (CNC, 2002) plantea que esta mutación es ininterrumpida, constante, rápida y tiene tendencia a acelerarse e influye en todo el planeta, en casi todas las esferas y condiciones de las actividades que se desarrollan en las organizaciones, así como en la vida del hombre y la sociedad.

Esa dimensión internacional mutante de los países, no es un fenómeno nuevo. Lo que ha variado es la intensidad y la extensión a través de la cual se manifiesta. Los países para responder con pertinencia, es decir satisfacer las necesidades del entorno, deben tener capacidad de adaptación, apertura a la innovación, flexibilidad en sus estructuras y sistemas. ya que el cambio siempre ha representado un proceso permanente $y$ continuo. Venezuela como parte del sistema mundial debe participar con calidad. No se puede negar la profunda crisis política actual, la cual repercute en lo económico, social, y tecnológico, entre otros.

Sin embargo, Acosta y Marval (2007), plantean que uno de los grandes retos que debe enfrentar Venezuela para insertarse en ese entorno internacional es la preparación del talento humano. Para lograrlo debe cerrar la brecha existente entre conocimiento y entrenamiento a objeto de participar en el espacio global. 
Se debe dar una reforma del sistema educativo, lo cual implica ir hacia la construcción e implementación de nuevos enfoques para abordar, analizar y actuar sobre la realidad. Para las autoras la competitividad del país depende fundamentalmente de la calidad del talento humano que posee.

Ante ese escenario, es evidente que la educación no puede estar al margen. El sistema educativo venezolano se ha visto obligado a considerar un panorama completamente distinto al presentado en décadas anteriores, lo cual le ha exigido emprender procesos de transformación en cada uno de sus niveles y modalidades y desarrollar una alta participación en la construcción de la sociedad. Para que el sistema educativo esté a la par de los avances científicos, tecnológicos y humanísticos, se han propuesto reformas, fundamentadas en un proceso de interacción constructiva y creativa del ser, el hacer, el conocer y el convivir entre los diferentes actores del proceso educativo.

Bajo ese contexto, se considera importante lo expuesto por Knight (2006), quien ha identificado varios problemas que requieren ser superados por las instituciones de educación superior latinoamericanas para estar en condiciones de responder a los desafíos presentados a escala mundial. En el primer nudo crítico está el tema del financiamiento estatal, el cual ha sido insuficiente en casi todas las instituciones universitarias de carácter público. En el segundo, refiere el autor la gestión universitaria, caracterizada por la implementación de formas de gobierno universitario nada adecuadas para generar lo denominado "liderazgo de cambio" dentro de las instituciones. El tercer gran problema está constituido por lo que él denomina la competencia global. En este sentido, argumenta que la universidad latinoamericana deberá enfrentar el desafío tanto en el ámbito interno como en el externo, pues existe un mundo donde la competencia de formación también está globalizada. De esa manera, la competencia ya no va a ser entre las instituciones universitarias de una región o de un país, sino que va a ser, cada vez más, una competencia global.

Para lograr desafiar esos retos las instituciones de educación superior deben concebirse como sistemas globales compuestos en su interior por subsistemas en interacción y con múltiples interacciones con su entorno social. Cada subsistema debe responder con calidad, pues de ello depende la excelencia de la institución.

Es innegable la importancia del personal administrativo en cualquier subsistema educativo, puesto que constituye el centro de apoyo a las actividades de docencia, investigación, extensión y producción de las universidades. Este personal debe tener las competencias necesarias para asumir los problemas propios de su área de trabajo y personal, y por ende cumplir eficientemente sus labores. Resulta paradójico entonces, que si todos los elementos en una organización son importantes, por qué en las instituciones educativas hay muy pocos escritos o investigaciones sobre la importancia del personal administrativo para el logro de los objetivos institucionales.

En ese sentido, transformar la práctica administrativa implica, necesariamente, perfilar un personal diferente, 
con nuevos conocimientos, métodos y técnicas cónsonas con el proceso de transformación e innovación característicos del momento. Un personal que, por supuesto, deberá actuar proactivamente ante el nuevo paradigma educativo y mundial (Hernández, 2005; Díaz y Márquez, 2005; Leal y Sánchez, 2006).

Una interrogante que se desprende de ese análisis es ¿cómo lograr perfilar ese personal? Una educación de calidad exige personal capaz de contribuir con la transformación del sistema educativo, para que este vaya a la par de los avances científicos, tecnológicos y humanísticos; entonces es indispensable, un entrenamiento continuo de ese personal a través de programas que cambien su manera de ser, pensar y actuar; ayudándoles a construir una nueva forma abierta al cuestionamiento y al crecimiento personal, a la crítica reflexiva, al diálogo y al desarrollo integral de sus propias potencialidades, ya que toda organización debe contar con personas con las competencias necesarias para actuar proactivamente en ese mundo global.

En el logro de ese reto, juega un papel fundamental e invaluable el proceso de entrenamiento del personal administrativo de las universidades, de allí la importancia que las instituciones de educación superior desarrollen y pongan en marcha programas de entrenamiento para dar posibilidad a su personal de ser protagonista de su formación en el marco de un proceso de reflexión y transformación de su práctica laboral. Esto implica que el contenido del programa de entrenamiento debe responder a sus propias necesidades y expectativas, debe ser dinámico y flexible con posibilidad de adap- tación de acuerdo con las circunstancias del presente y del futuro. El propósito de esos programas consiste en mejorar las habilidades relacionadas con las tareas específicas de los miembros del personal, deben basarse en competencias (Canquiz, 2003, Castejón y Zamora, 2001).

La primera etapa a desarrollar para implementar esos programas es realizar un estudio para detectar las necesidades de entrenamiento. Esta es una de las etapas más complejas de este proceso, cuyo objetivo es investigar cuáles son las necesidades reales y prioritarias a satisfacer, traduciéndolas en conocimientos, habilidades, actitudes y aptitudes que deberán ser adoptadas, desarrolladas o modificadas para mejorar la calidad del trabajo y la prestación integral del personal. Esta detección implica realizar un diagnóstico de las carencias reales de alguna competencia para que el trabajador pueda conocer y contrarrestar esas debilidades y cumplir eficientemente una función o tarea.

En ese marco de ideas, se encuentra la Universidad Nacional Experimental Rafael María Baralt (UNERMB), la cual no cuenta con un programa de entrenamiento de su personal administrativo. A través de los diferentes Programas Académicos y de la Unidad de Personal se organizan cursos de capacitación pero sin responder a un diagnóstico real de necesidades de entrenamiento. Sólo se aplica un instrumento para identificar los cursos que desea realizar el personal, pero sin considerar las competencias requeridas para mejorar su desempeño.

Por lo tanto, el desconocimiento de estas necesidades reales ha traído como 
consecuencia la apertura de actividades de entrenamiento no acordes con la exigencia de los cargos y en muchas oportunidades los empleados han realizado cursos, talleres o cualquier otra actividad de aprendizaje que verdaderamente no necesita. Esto genera en ellos desmotivación y descontento, puesto que se desconocen los criterios utilizados para la selección de las personas a capacitar.

De allí que el propósito de esta investigación es detectar las necesidades de entrenamiento del personal administrativo que ocupa cargo profesional en el área de finanzas de la UNERMB, como primera etapa para el diseño de un programa de entrenamiento con base en competencias. Es importante mencionar que la UNERMB fue creada en marzo de 1982 y está ubicada en la Sub-región Costa Oriental del Lago de Maracaibo (COLM) en el estado Zulia, Venezuela.

La investigación es de tipo descriptiva, con un diseño no experimental, de campo, univariable y de acuerdo a la fuente de información es mixto pues se utilizó fuente primaria y secundaria. La población está constituida por el personal administrativo profesional de la UNERMB que se desempeña en el área de las finanzas. Según el Manual de la Oficina de Planificación del Sector Universitario (OPSU), los cargos están ubicados en determinados grupos como se muestra en la Tabla 1.

Se utilizó la técnica de revisión documental y la encuesta. Se aplicó a la población, un cuestionario semiestructurado con 58 ítems y tres (3) alternativas de respuestas: siempre, algunas veces y nunca; y con dos (2) preguntas abiertas con la finalidad de dar flexibilidad al ins-

\section{Tabla 1}

Personal administrativo de la UNERMB (población)

\begin{tabular}{lc}
\hline \multicolumn{1}{c}{ Grupo } & $N^{\circ}$ de cargos \\
\hline Contaduría & 5 \\
Presupuesto & 6 \\
Administración & 31 \\
Análisis y Control Financiero & 3 \\
Control Interno y Auditoria & 4 \\
Total & 49 \\
\hline
\end{tabular}

Fuente: Unidad de Personal de la UNERMB.

trumento. Se aplicó la validez de contenido, a través del juicio de expertos. Para la confiabilidad se aplicó una prueba piloto y el coeficiente de confiabilidad de AlfaCronbach, cuyo resultado es de 0,96.

\section{Entrenamiento, capacitación, adiestramiento y formación ¿términos divergentes o convergentes?}

En esta investigación, se asume la concepción de entrenamiento manejada por Reyes (1997), por considerar que plantea una visión más amplia del término englobando a la capacitación, el adiestramiento y a la formación, como tres (3) aspectos distintos y complementarios, los cuales se describen a continuación:

1. La capacitación, de carácter más bien teórico, de amplitud mayor y para trabajos calificados.

2. El adiestramiento, de carácter más práctico y para un puesto concreto; es necesario en toda clase de trabajos, e indispensable, aún suponiendo una capacitación previa. El término adiestramiento es muy exacto, significa 
"adquirir destrezas", es decir, se adquiere la formación de hábitos por el ejercicio sistemáticamente realizado, de manera semejante se adquiere facilidad, precisión y rapidez en el desarrollo de un trabajo por medio de una enseñanza práctica y de carácter sistemático.

3. La formación, se requiere para crear o desarrollar en el obrero o empleado, pero sobre todo en el jefe "hábitos morales, sociales, de trabajo", entre otros, que no pueden darse en la capacitación o adiestramiento y sin embargo, son indispensables para que el trabajador sea leal, sereno, ordenado y decidido.

Las tres (3) funciones antes mencionadas, de acuerdo con los planteamientos de Reyes (1997), se requieren para todos los puestos; pero evidentemente si en los niveles inferiores no calificados predomina el adiestramiento, conforme se asciende en los niveles jerárquicos, tiene mayor importancia la capacitación y la formación.

\section{Detección de necesidades: fase inicial del proceso de entrenamiento}

En este estudio las investigadoras conciben la etapa detección de necesidades según los postulados de Chiavenato
(2002, 2004), como inventario de necesidades de entrenamiento o diagnóstico. Se describe detalladamente sólo la primera fase descrita por el autor, la cual se asume para sustentar el proceso de investigación, así como algunos elementos planteados por Hill, Estrada y Bosch (2003). El Inventario de necesidades de entrenamiento, corresponde al primer diagnóstico preliminar de lo que debe hacerse. Puede efectuarse en tres (3) niveles de análisis: (a) análisis de la organización total, (b) análisis de los recursos humanos y (c) análisis de las tareas.

Según los aportes de Chiavenato (2004), una necesidad de entrenamiento es una diferencia entre los requisitos exigidos por el cargo y las habilidades actuales del ocupante del mismo. Es importante resaltar que como en esta investigación se aborda el enfoque de competencias, las necesidades de entrenamiento están determinadas por la relación expresada en el Diagrama 1.

\section{Concepción de competencias}

En esta investigación se asume la definición de competencias de Benavides (2002: 72), entendida como "comportamientos y destrezas visibles que la persona aporta en un empleo para cumplir con sus responsabilidades eficaz y satisfacto-

\section{Diagrama 1 \\ Necesidades de entrenamiento}

Competencias que requiere desarrollar el ocupante del cargo
Competencias actuales del ocupante del cargo
Necesidades de entrenamiento

Elaboración propia. 
riamente". Las considera como comportamientos observables manifiestos que la persona aporta en un empleo y están relacionados con la efectividad de su desempeño en la práctica laboral.

Según la referida autora las competencias engloban no sólo las aptitudes, sino también los factores individuales como: (a) conocimientos; (b) habilidades y destrezas; (c) representaciones mentales o significados del trabajo y de sí mismo; (d) rasgos y temperamento; (e) motivos y necesidades; y (f) capacidad de adecuación, generación y aplicación. Estos elementos son considerados por la autora como "fundamentos" de las competencias, es decir, si el individuo carece de conocimientos requeridos para una actividad laboral, se hace imposible cumplir a cabalidad con sus responsabilidades, más sin embargo, el hecho que los posea no significa que los aplique.
No obstante, según el Modelo Iceberg existen competencias fáciles de observar y otras que son difíciles (Gráfico 1). Para Spencer y Spencer (2003), "EI conocimiento y las habilidades generalmente son visibles y relativamente superficiales a las personas. El autoconcepto, los rasgos de personalidad y las motivaciones son aspectos más ocultos, profundos y centrales de las personas". Para estos autores el conocimiento y las habilidades son más fáciles de desarrollar.

En concordancia con las ideas expuestas, Santos (2007) asume que las competencias difíciles de observar son la base de la selección de personal y las visibles constituyen, por otro lado, la clave de la política de desarrollo. Es preciso señalar que compartimos las ideas expuestas por los autores, porque realmente hay comportamientos difíciles de observar, y a la hora de llevar a cabo un proceso de

\section{Gráfico 1}

Modelo iceberg. Competencias para el trabajo. Modelos para un rendimiento superior (2003)

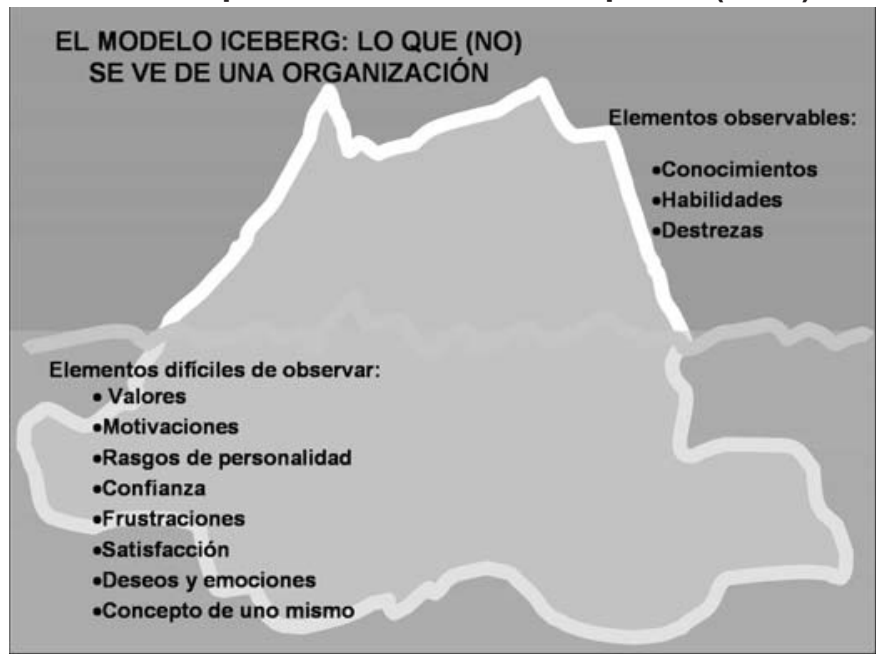

Fuente: Spencer L. y Spencer, S. Adaptado por Acosta, Marval y Revilla (2004). 
entrenamiento, éste debe considerar estrategias acordes con estas apreciaciones, enfatizando en el desarrollo y aprendizaje de las competencias ocultas en el individuo.

De acuerdo a Benavides (2002), las competencias se clasifican en: (a) genéricas, (b) laborales y (c) básicas.

Las competencias genéricas: son características requeridas por los individuos que pueden generalizarse en una organización. Éstas nacen de las políticas y los objetivos de la organización y su finalidad es fortalecer la identidad. Son consideradas cualidades o atributos directamente relacionados con el desempeño en grupos de empleos determinados y afines; no son generalizables entre organizaciones, áreas o niveles organizacionales.

A continuación se presenta una lista de competencias genéricas establecida por Woodruffe (citado en Benavides, 2002), de las cuales pueden seleccionarse y aplicarse las que se consideren pertinentes de acuerdo a la singularidad de cada organización: (a) amplitud de conocimientos para estar bien informado, (b) astucia para tener un entendimiento claro, (c) razonamiento para encontrar alternativas, (d) organización para trabajar productivamente, (e) se enfoca en conseguir resultados, (f) liderazgo, (g) sensibilidad para identificar otros puntos de vista, (h) cooperación para trabajar en equipo e (i) orientación a conseguir objetivos a largo plazo.

Las competencias laborales son los atributos personales visibles o comportamientos que se aportan al trabajo para lograr un desempeño idóneo y eficiente. Estas competencias además Integran elementos característicos del ser humano tales como: (a) conocimientos, (b) habilidades, (c) autoconcepto, (d) rasgos y temperamento y (e) motivos y necesidades.

Así mismo, concibe las competencias básicas como un grupo de competencias mínimas y comunes a los diferentes sectores laborales, áreas y niveles organizacionales. Son consideradas como los cimientos que fundamentan y posibilitan el desempeño laboral satisfactorio y al mismo tiempo sustentan el aprendizaje ocupacional. Hacen referencia a la capacidad del individuo para transferir en su vida cotidiana la información, conocimientos, aptitudes y habilidades desarrolladas en la escuela básica. La autora, las clasifica en tres (3) grupos:

1. Habilidades básicas: capacidad lectora, escritura, aritmética, matemáticas, hablar y escuchar

2. Desarrollo del pensamiento: pensamiento creativo, solución de problemas, toma de decisiones, asimilación y comprensión, capacidad de aprender a aprender y razonar (organizar conceptos).

3. Cualidades personales: autorresponsabilidad, autoestima, sociabilidad, autodirección, integridad.

\section{Análisis de los resultados}

Para el análisis de los resultados de la investigación se utilizaron los siguientes intervalos (Tabla 2).

El baremo para los diferentes tipos de competencias se muestra en las Tablas 3, 4 y 5. La dimensión competencias genéricas consta de 32 ítems, por lo tanto, el límite inferior es 32 y el máximo es 96 (Tabla 3). 
Las competencias laborales están constituidas por 15 ítems, resultando como baremo el presentado en la Tabla4 .

El baremo de las competencias básicas se mide a través de 11 ítems (Tabla 5).

Para efecto de la presentación de los resultados en este artículo solo se incluirán las tablas y gráficos con los datos obtenidos de la aplicación del cuestionario Detección de Necesidades de Entrenamiento (DNE) correspondientes al primer grupo del personal administrativo (Contaduría), por lo extenso de los mismos.

\subsection{Análisis de las competencias que posee el Grupo Contaduría}

A continuación se muestran los datos con relación al nivel de competencias detectado para el Grupo Contaduría.

- Competencias genéricas: los resultados presentados revelan que el $63 \%$, en promedio tiene un nivel de competencia mediano.

- Competencias laborales: se observa en el Cuadro 1 , que el $51 \%$ del grupo de Contaduría, posee medianamente esta competencia.

- Competencias básicas: el Grupo de Contaduría se adjudica un $67 \%$ de las competencias básicas, es decir, las desarrollan medianamente, tal como se observa en el Cuadro 2.

Es importante resaltar que una vez analizadas las competencias genéricas, laborales y básicas del grupo de Contaduría, los resultados indican que en todas prevalece la categoría mediana. Este hallazgo revela que el personal cuenta con competencias para desarrollar su trabajo,
Tabla 2

Baremo para la variable necesidades de entrenamiento

\begin{tabular}{ccc}
\hline Puntuación & Rangos & Categorías \\
\hline $58-96$ & III & Baja \\
$97-135$ & $\|$ & Mediana \\
$136-174$ & I & Alta \\
\hline
\end{tabular}

Fuente: Elaboración propia.

Tabla 3

Baremo competencias genéricas

\begin{tabular}{ccc}
\hline Puntuación & Rangos & Categorías \\
\hline $32-53$ & III & Baja \\
$54-75$ & $\|$ & Mediana \\
$76-96$ & I & Alta \\
\hline
\end{tabular}

Fuente: Elaboración propia.

Tabla 4

Baremo competencias laborales

\begin{tabular}{ccc}
\hline Puntuación & Rangos & Categorías \\
\hline $15-25$ & III & Baja \\
$26-36$ & II & Mediana \\
$37-45$ & I & Alta \\
\hline
\end{tabular}

Fuente: Elaboración propia.

Tabla 5

Baremo competencias básicas

\begin{tabular}{ccc}
\hline Puntuación & Rangos & Categorías \\
\hline $11-18$ & III & Baja \\
$19-26$ & II & Mediana \\
$27-33$ & I & Alta \\
\hline
\end{tabular}

Fuente: Elaboración propia.

sin embargo es necesario considerar los aspectos donde se detectaron debilidades para ejercer eficientemente su cargo, con la finalidad de diseñar un programa de entrenamiento que atienda de manera específica tal situación. 


\section{Cuadro 1 \\ Competencias laborales que posee el Grupo Contaduría}

Competencias laborales

EXI. NO N AV

$\mathrm{S}$

EXI. (\%)

(\%) (\%)

\section{* Conocimientos}

Aplicar los conocimientos teóricos adquiridos en su formación $\quad X$

$\begin{array}{llll}X & 0 & 80 & 20 \\ X & 0 & 40 & 60\end{array}$

Aplicar los conocimientos prácticos adquiridos en su 60 formación profesional para desempeñarse en el cargo que ocupa

Utilizar el aprendizaje obtenido de experiencias anteriores para desempeñarse

en su cargo

\section{* Habilidades}

Realizar operaciones matemáticas relacionadas con su área $x$

$\begin{array}{cccc} & 0 & 20 & 80 \\ \times & 60 & 20 & 20 \\ & & & \\ X & 0 & 60 & 40 \\ X & 0 & 60 & 40\end{array}$

Dominar los lineamientos para la elaboración de informes técnicos

Analizar los informes técnicos

Utilizar los sistemas contables que existen en la actualidad

\section{* Destrezas}

Manipular el equipo de computación para realizar su trabajo $X$

Manejar los programas de computación requeridos para

$\mathrm{X}$

ejercer su cargo

Manejar las máquinas calculadoras científicas relacionadas

$X$

con el área administrativa

\section{* Autoconcepto}

Seguridad en cada una de las actividades que realiza

Compartir los éxitos laborales obtenidos con sus compañeros de trabajo

Compartir los fracasos laborales obtenidos con sus

compañeros de trabajo

Contribuir con las metas organizacionales

$\begin{array}{llll}X & 0 & 40 & 60 \\ X & 0 & 60 & 40\end{array}$

Cumplir con las expectativas del cargo

$\begin{array}{rrrr}X & 0 & 20 & 80 \\ X & 0 & 40 & 60\end{array}$

Categorías de competencias laborales

Fuente: Elaboración propia. 


\section{Cuadro 2}

Competencias básicas que posee el Grupo Contaduría

\begin{tabular}{cccccc}
\hline Competencias básicas & EXI. & NO & $\mathrm{N}$ & AV & $\mathrm{S}$ \\
& & EXI. & $(\%)$ & $(\%)$ & $(\%)$ \\
\hline
\end{tabular}

\section{* Habilidades básicas}

Comprender los informes técnicos que recibe de otras instancias de la Universidad

Redactar informes

Presentar en público las metas cumplidas en su Departamento

$\times \quad 40$

$\begin{array}{cccc} & & 60 & 0 \\ \times & 0 & 100 & 0 \\ \times & 60 & & \\ & & 40 & 0\end{array}$

* Desarrollo del pensamiento

Aplicar técnicas para diseñar formatos, elaborar informes relacionados con su trabajo

Consultar con especialistas para decidir la alternativa de solución frente a un problema

X 0

$80 \quad 20$
$\times-20=0$

X 20

$40 \quad 40$

Analizar las causas y las alternativas de solución para poder $\quad \mathrm{X}$ elegir la decisión más acertada y favorable

$60 \quad 20$

Participar en actividades de aprendizaje (cursos, talleres, seminarios u otra actividad) relacionados con su trabajo

\section{* Cualidades personales}

Elaborar una agenda que le facilite cubrir las actividades para obtener los objetivos planificados

Participar con otras personas a fin de promover relaciones interpersonales

Realizar actividades que orienten el mejoramiento continuo en sus labores diarias

Promover actividades sociales que estimulen la participación de sus compañeros de trabajo $\mathrm{X} \quad 0$

Categorías de competencias básicas

Baja Mediana Alta 15 67

Fuente: Elaboración propia.

\subsection{Detección de las necesidades de entrenamiento para el desarrollo de competencias. Grupo Contaduría}

Competencias genéricas: de acuerdo con el análisis de las competencias que posee el Grupo Contaduría se detectó que el grado de necesidad para desarrollar este tipo de competencia se ubica en la categoría mediana al obtener un puntaje definitivo de 60 , tal como se puede visualizar en el Gráfico 2.

Para interpretar el gráfico, se parte del hecho de que si todos los empleados que conforman este grupo hubiesen respondido que siempre realizan las activi- 
dades señaladas en el cuestionario DNE, la puntuación resultante sería 32 , esto significa que si el resultado obtenido se acerca a este número disminuye el grado de necesidad.

Según los resultados, el $75 \%$ del grupo de Contaduría requiere desarrollar competencias en las siguientes actividades: desarrollo de canales de comunicación, acceso a la Internet, análisis de problemas, prever recursos, identificación de actividades prioritarias, implementación de nuevas formas de trabajo, organización de las tareas antes del plazo límite, liderazgo, elaborar propuestas de procedimientos, ofrecer nuevas ideas para realizar el trabajo en los departamentos, sensibilidad para identificar otros puntos de vista, cooperación para trabajar en equipo y orientación a conseguir objetivos a largo plazo, entre otros.

Competencias laborales: al igual que en la anterior, la necesidad de entrenamiento del grupo de Contaduría en este tipo de competencias se ubica en la categoría mediana, tal como se muestra en el Gráfico 3.

Para cerrar la brecha existente es necesario que el $63 \%$ de los empleados reciban entrenamiento. Del análisis realizado sobre las competencias laborales actuales que tiene este Grupo, expresadas en la Tabla 3, se desprenden las actividades que deben desarrollar para ser competentes, las cuales se mencionan a continuación: aplicación de los conocimientos teóricos, dominio de los lineamientos para elaborar informes técnicos, análisis de informes técnicos, manejo de máquinas calculadoras relacionadas con el área administrativa, manejo de equipos

\section{Gráfico 2 \\ Necesidades de entrenamiento para el desarrollo de competencias genéricas del Grupo de Contaduría}

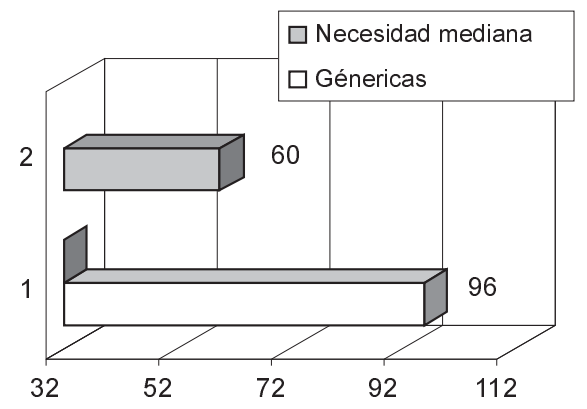



de computación y de los programas requeridos para ejercer los cargos, y desarrollo de autoconcepto.

Competencias básicas: al igual que en las competencias genéricas y laborales la necesidad de entrenamiento del grupo de Contaduría en este tipo de competencias se ubica en la categoría mediana, en el Gráfico 4 se visualiza lo planteado. 


\section{Gráfico 4 \\ Necesidades de entrenamiento para el desarrollo de competencias básicas del Grupo de Contaduría}

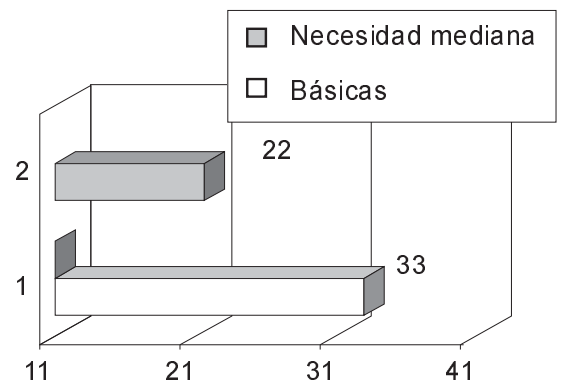

Para desarrollar este tipo de competencia el personal administrativo debe realizar actividades como: redacción y comprensión de informes técnicos, promoción de actividades sociales que estimulen la participación de todos, análisis de causas y alternativas de solución para la toma de decisiones, participación en actividades de aprendizaje como cursos, talleres, seminarios u otros relacionados con su trabajo y promoción de relaciones interpersonales, entre otros.

\subsection{Análisis de las competencias que posee el Grupo Presupuesto}

Competencias genéricas: al analizar el grupo de Presupuesto se observó que el énfasis de las respuestas obtenidas de la aplicación del instrumento está en la alternativa algunas veces, pues así lo manifestó el $53 \%$ de los encuestados. Los datos evidencian que los empleados algunas veces toman en cuenta los puntos de vista de otros o atienden las expectativas de sus compañeros de trabajo, a pesar de no ser un requisito exigido por el
Manual. Mientras el $83 \%$ de los mismos indicó asistir algunas veces a cursos de capacitación como una forma de ampliar sus conocimientos, seguir un plan de acción para el logro de las metas o elaborarlo con la finalidad de determinar el personal requerido para el logro de los objetivos, ésta última es una condición exigida para ejercer el cargo.

Asimismo, el $67 \%$ expresó que algunas veces proponen procedimientos para asegurar resultados favorables, implementan técnicas para analizar en grupo las alternativas de realización de las labores, trabajan en equipo e involucran a otras personas en su área para lograr los objetivos, aun cuando las dos primeras de las mencionadas no se les exige que las cumplan.

Competencias laborales: al igual que las genéricas, el $50 \%$ de los entrevistados han desarrollado medianamente esta competencia.

Competencias básicas: también predomina la categoría mediana con un $48 \%$ ubicándose en la alternativa algunas veces.

\subsection{Detección de las necesidades de entrenamiento para el Grupo Presupuesto}

Competencias genéricas: se detectó que la necesidad para el desarrollo de este tipo de competencia se posiciona en la categoría mediana al obtenerse un puntaje definitivo de 59. Para atender estas necesidades de entrenamiento del grupo en referencia, se deben atender prioritariamente lo siguiente: asistencia a cursos de capacitación para ampliar sus conocimientos y habilidades, acceso a la 
Internet, establecimiento de un plan de acción donde se elabore un cronograma sobre el tiempo, personal y los recursos materiales requeridos para lograr los objetivos y metas organizacionales, implementación de técnicas para analizar en grupo las alternativas de realización de labores, tomar en cuenta los puntos de vista y expectativas de sus compañeros en cuanto al cargo.

Competencias laborales: la necesidad para el desarrollo de este tipo de competencia se posiciona en la categoría mediana con un puntaje de 62. Para reforzar estas competencias se debe hacer énfasis en el desarrollo de las siguientes actividades: manejo de programas de computación y máquinas calculadoras científicas, manipulación de equipos de computación, dominio de los lineamientos para elaborar informes técnicos, análisis de informes técnicos y actualización en sistemas contables, entre otros.

Competencias básicas: se ubica, al igual que las competencias genéricas y laborales en la categoría mediana, al obtener un puntaje de 72 . Con relación al desarrollo de actividades para cubrir las necesidades de entrenamiento del grupo de Presupuesto en las competencias básicas, es importante resaltar que coinciden con las necesidades de entrenamiento detectadas en el grupo de Contaduría.

\subsection{Análisis de las competencias que posee el Grupo Administración}

Competencias genéricas: este grupo está conformado por el mayor número de personal del área de finanzas. Al igual que los anteriores, sus respuestas se concentraron en la alternativa algunas veces, prevaleciendo también la categoría mediana con un $56 \%$ de los informantes clave.

Competencias laborales: una vez analizados tres de los grupos que conforman el área de financiera, los resultados obtenidos mantienen cierta similitud en relación a que todos se ubican en la categoría mediana, sin embargo, en la competencia laboral, el $45 \%$ del grupo de Administración posee competencias alta, le sigue un $43 \%$ con competencia mediana; aspecto importante puesto que el Manual exige que desarrollen cada una de las competencias contempladas en los indicadores referidos a conocimientos, habilidades y destrezas, sin embargo, se observó que las más importantes, como utilizar los sistemas contables actuales, manejar los programas de computación, manipular la computadora y máquinas calculadoras, realizar operaciones matemáticas y analizar informes técnicos, un número considerable sólo algunas veces lo hace.

Competencias básicas: también predominan las competencias medianas con un $41 \%$. A pesar que los indicadores seleccionados para evaluarla, son exigidos por el Manual de cargos de la OPSU, como: habilidades básicas, desarrollo del pensamiento y cualidades personales, un $54 \%$ expresó que nunca presentan ante otra instancia las metas cumplidas en su departamento, el $46 \%$ no diseña formatos para presentar informes técnicos, y el $57 \%$ nunca participa en actividades de aprendizaje para mejorar su desempeño, como: cursos, seminarios u otra actividad. Además, se nota poca participación en las actividades inherentes al indicador cualidades personales. 


\subsection{Detección de las necesidades de entrenamiento para el desarrollo de competencias. \\ Grupo Administración}

Competencias genéricas: el mayor porcentaje se localiza en las alternativas siempre y algunas veces con un total de $75 \%$ entre ambas, por lo cual estas quedan ubicadas en el rango 2 del baremo correspondiente, categoría mediana.

El Grupo de Administración para desarrollar las competencias genéricas requiere realizar las siguientes actividades: desarrollo de canales de comunicación, implementación de técnicas para analizar en grupo la realización de las labores, asistencia a cursos para ampliar sus conocimientos y habilidades, acceso a la Internet, análisis de alternativas de solución a problemas, implementación de nuevas formas de trabajo, implementación de técnicas para analizar en grupo elaborar propuestas de procedimientos para asegurar resultados favorables, ofrecer ideas para realizar el trabajo en su departamento.

Competencias laborales: su necesidad de entrenamiento se ubica en la categoría baja, rango III del baremo, ya que en las alternativas de respuestas de los encuestados el mayor porcentaje se adjudicó a siempre con un $55 \%$.

Competencias básicas: el mayor porcentaje se presenta en las alternativas nunca y algunas veces con un total de $76 \%$ entre ambas, por lo cual su lugar de ubicación es el rango II, en la categoría mediana. La ejecución de actividades debe estar orientada hacia el desarrollo de la habilidad para redacción y comprensión de informes técnicos; análisis de causas y alternativas de solución para toma de decisiones; participación en cursos, seminarios, talleres relacionados con su trabajo; relaciones interpersonales y participación y promoción de actividades sociales, entre otras.

\subsection{Análisis de las competencias que posee el Grupo Análisis y Control de Finanzas}

Competencias genéricas: este grupo se distingue del resto porque a pesar de que el mayor porcentaje se ubica en la categoría de competencia mediana $(53 \%)$, las respuestas emitidas por los empleados se concentran en la alternativa de respuesta siempre con un $100 \%$, lo cual guarda una estrecha relación con lo exigido por el Manual para desempeñar el cargo y la actividad que ellos realizan. De allí que sobresale que en cada uno de estos indicadores: amplitud de conocimientos, astucia para tener un entendimiento claro, razonamiento para encontrar alternativas, organización para trabajar productivamente, liderazgo, sensibilidad para identificar otros puntos de vista y cooperación para trabajar en equipo, el $100 \%$ respondió que siempre lo hacen.

Competencias laborales: es el segundo grupo cuya categoría de competencia laboral es alta con un $54 \%$ del total, de los cuales el $100 \%$ respondió que siempre realizan actividades para desarrollar los conocimientos teóricos y prácticos y desempeñarse en el cargo, además, poseen la habilidad para realizar operaciones matemáticas, manejan las máquinas calculadoras científicas y contribuyen al logro de las metas organizacionales, entre otras. 
Competencias básicas: en este grupo el $28 \%$ de los encuestados se ubica en un nivel de competencia alto y un $36 \%$ en mediano.

\subsection{Detección de necesidades de entrenamiento para el desarrollo de competencias. Grupo Análisis y Control de Finanzas.}

Competencias genéricas: en estas el mayor porcentaje recae en las alternativas siempre y algunas veces, con un total de $56 \%$ entre ambas, respondiendo a una ubicación en la categoría de necesidad de entrenamiento baja. Esto significa que este grupo presenta fortalezas en la realización de las actividades inherentes el desarrollo de estas competencias.

Competencias laborales: se ubicaron en el rango III, categoría baja. Se debe destacar que el comportamiento de estas competencias es similar a las laborales del Grupo Administración puesto que el mayor porcentaje obtenido se presenta para las alternativas: algunas veces $33 \%$ y siempre un $54 \%$. Esto quiere decir que para el personal administrativo de este grupo, la categoría de necesidad de entrenamiento es baja, equivalente al rango III del baremo.

Competencias básicas: el mayor porcentaje se presenta en las alternativas nunca y algunas veces con un total de $72 \%$ entre ambas, por lo cual su lugar de ubicación es en la categoría mediana, rango II. La ejecución de actividades debe estar orientada hacia el desarrollo de la habilidad para redacción y comprensión de informes técnicos. También hacia la aplicación de técnicas para diseño de formatos y elaboración de informes rela- cionados con su trabajo; participación en cursos, seminarios, talleres; planificación de actividades para obtener objetivos, relaciones interpersonales y participación y promoción de actividades sociales que estimulen a sus compañeros, entre otras.

\subsection{Análisis de las competencias que posee el Grupo Control Interno y Auditoría}

Competencias genéricas: a este grupo el Manual les demanda para el desempeño del cargo, desarrollar la competencia en cada uno de los indicadores establecidos, a excepción del indicador organización para trabajar productivamente donde no se le exige cumplir ninguna actividad. La categoría de competencia es mediana, el $75 \%$ algunas veces desarrolla canales de comunicación, ofrece ideas nuevas al resto del grupo y atienden a sus recomendaciones, mientras el $100 \%$ informó que sigue el plan de acción para lograr las metas organizacionales.

Competencias laborales: al igual que los grupos Administración y Análisis y Control de Finanzas, la categoría de competencia es alta, ubicada en $57 \%$. Aquí el $100 \%$ manifestó que siempre desarrollan competencias relacionadas con los conocimientos, las habilidades y destrezas que este grupo debe desarrollar al igual que el autoconcepto, entre las cuales se destacan: la aplicación de conocimientos prácticos para desempeñarse eficazmente, así como realizar operaciones matemáticas relacionadas con el área.

Competencias básicas: se ubica en la categoría mediana al igual que en los demás grupos. A pesar que el Manual le 
exige analizar las causas y las alternativas de solución para poder elegir la decisión más acertada y favorable, como asistir a cursos, el $25 \%$ expresó que nunca lo hace. Mientras el $50 \%$ algunas veces, redactan informes y promueven las relaciones interpersonales.

\subsection{Detección de las necesidades de entrenamiento para el desarrollo de competencias. Grupo Control Interno y Auditoría.}

Competencias genéricas: de acuerdo a los porcentajes obtenidos el mayor se ubica entre las alternativas siempre y algunas veces con un $63 \%$ en total para ambas, el cual es equivalente a la frecuencia de realización de las actividades expresadas por los encuestados, por lo que estas quedan ubicadas en el rango Il del baremo presentado, en la categoría de necesidad de entrenamiento mediana.

Por lo planteado anteriormente se debe hacer énfasis en las actividades que contribuyan a desarrollar estas competencias: asistencia a cursos como forma de ampliar conocimientos; acceso a la Internet para mantenerse informados; análisis de variables y planteamiento de alternativas para resolución de problemas; diseño de planes de acción para el logro de los objetivos propuestos; propuestas de procedimientos adecuados en situaciones problemáticas; y seguimiento de un plan de acción para lograr las metas organizacionales.

Competencias laborales: se ubica en el rango III del baremo, categoría baja, en las alternativas de respuestas el mayor porcentaje: $57 \%$, correspondió a siempre, por lo tanto se deduce que este grupo presenta fortalezas en la realización de las actividades inherentes al desarrollo de estas competencias.

Competencias básicas: el mayor porcentaje se ubica en las alternativas nunca y algunas veces con un total de $77 \%$ entre ambas, por lo cual su lugar de ubicación es en el rango Il, categoría mediana. La ejecución de actividades debe estar orientada hacia el desarrollo de la habilidad para redacción y comprensión de informes técnicos; aplicación de técnicas para diseño de formatos; análisis de causas y alternativas de solución para toma de decisiones; participación en actividades de aprendizaje como: cursos, seminarios, talleres relacionados con su trabajo; relaciones interpersonales y participación y promoción de actividades sociales, entre otras.

Del diagnóstico realizado sobre la detección de necesidades de entrenamiento del personal administrativo del área de finanzas de la UNERMB, los resultados revelaron la existencia de un grado de necesidad de entrenamiento mediana; lo que significa Para contribuir con el desarrollo de las competencias del personal, consideran que una manera de hacerlo es a través de actividades educativas, como lo manifestaron en sus respuestas a las dos (2) preguntas abiertas formuladas en el cuestionario DNEC.

\section{Conclusiones}

El instrumento utilizado para la detección de necesidades, se considera pertinente, pues permite tener una visión integral de la variable objeto de estudio. Se puede detectar las necesidades por 
persona, por estrato y para la población total, en función de los tipos de competencias: genéricas, laborales y básicas.

Los resultados obtenidos implican un cambio en la manera como la Universidad ha llevado a cabo el proceso de entrenamiento del personal administrativo, para lo cual es necesario tomar en cuenta aspectos importantes como: (a) reconocimiento de las competencias que permitan un alto desempeño y de las cuales carece ese personal (b) evaluación del nivel de competencia que posee la persona en comparación con el deseado, (c) comparación del proceso de entrenamiento tradicional y el proceso de entrenamiento por competencia. Todo esto permitirá elaborar programas de entrenamiento acorde con las necesidades detectadas.

Asimismo, el grupo de profesionales del área de finanzas al conocer las carencias en las competencias detectadas, sean genéricas, laborales y básicas, emprendan un proceso de desaprender y aprender hábitos de pensamientos, sentimientos y acción a manera de autoevaluar su desempeño, tomando en cuenta que cada persona es responsable de su proceso de aprendizaje. Ello les permitirá identificar sus necesidades de entrenamiento para darlas a conocer a sus jefes inmediatos con el propósito de establecer en conjunto, planes de acción que permitan un desempeño eficiente.

Los resultados de la investigación visualizan la necesidad de continuar con procesos de investigación para dar continuidad a este estudio, es decir, es importante detectar las necesidades en otro segmento del personal administrativo y emprender planes de entrenamiento que aporten soluciones a la situación detectada a corto y mediano plazo.

\section{Referencias bibliográficas}

Acosta, Ivonne. y Marval, Elina (2007). Evaluación institucional estratégica. Revista Anales. Vol.7. No. 1. Nueva serie. Venezuela, Universidad Metropolitana, pp 117-136.

Benavides, Olga. (2002). Competencias y competitividad: diseño para organizaciones latinoamericanas. Bogotá: Mc Graw Hill.

Canquiz, Liliana. (2003). Propuesta teórico metodológica para diseñar y evaluar perfiles profesionales. Proyecto de Tesis Doctoral no publicado. Maracaibo: La Universidad del Zulia.

Castejón, Heriberta y Zamora, Maigualida (2001).Perfil de competencias del grupo participante en el programa de eficiencia personal y laboral (EPYL). Revista Omnia. Año 7. Nos. 1 y 2. Venezuela. Universidad del Zulia, pp. 69-88.

Comisión Nacional de Currículo (2002). Lineamientos para abordar la transformación en la educación superior. Escenarios curriculares. V Reunión Nacional de Currículo para la Educación Superior. Caracas: UCV.

Chiavenato, Idalberto (2002). Gestión del talento humano. El nuevo papel de recursos humanos en las organizaciones. Colombia: McGraw Hill.

Chiavenato, Idalberto (2004). Administración. Proceso administrativo. Colombia: McGraw Hill.

Díaz, Judith y Márquez, Jeannette (2005). Formación del recurso humano: visión tradicional y visón por competencias. Revista Omnia. Año 11. No.2. Venezuela. Universidad del Zulia, pp. 7592. 
Hernández, Sergio (2005). Administración, pensamiento, proceso, estrategia y vanguardia. México: Mc Graw Hill.

Hill, María, Estrada, Samuel y Bosch, Zuyin. (2003). Formación, capacitación, desarrollo del recurso humano y su importancia en las organizaciones. Recuperado el 15 de julio de 2003 de www.monografías.com

Knight. Peter (2006). El profesorado de educación superior. Formación para la excelencia. España: Narcea, S.A De Ediciones.

Leal, Raiza y Sánchez, Ninoska (2006). Modelo de evaluación del desempeño basado en competencias para el personal de nivel operativo empresarial. Revista Universitas 2000. Vol.30. Venezuela, pp. 15-33.

Oficina de Planificación del Sector Universitario. Manual Descriptivo de Cargos Administrativos. Recuperado el 23 de junio de 2004 de http://www.adm. ula.ve/ personal/varios_opsu/presen taci\%f3n_opsu.htm

Reyes, Agustín (1997). Administración de personal. Relaciones humanas. México: Editorial Limusa.

Santos, Yaniel (2007). La gestión de competencias con enfoque por procesos. Recuperado el 22 de julio de 2007 de http://www.gestiopolis.com/organizacion-talento/gestion-por-competencias-con-enfoque-de-procesos.htm

Spencer, Lyle. y Spencer, Signe (2003). Competencias para el trabajo. Modelos para un rendimiento superior. $\mathrm{Re}$ cuperado el 25 de noviembre de 2003 de http://www.diba.es/fl/fitxers/pfm057esp.PDF.

Universidad Nacional Experimental "Rafael María Baralt" (2002). Plan para la transformación académica de la Universidad Nacional Experimental Rafael María Baralt. Cabimas. 